Please do not remove this page

RMIT

UNIVERSITY

\title{
Screening for the efficacy on lipid accumulation in 3T3-L1 cells is an effective tool for the identification of new anti-diabetic compounds
}

Zeng, Xiaoyi; Zhou, Xiu; Xu, Jun; Chan, Stanley Ming Hang; Xue, Charlie Changli; Molero - Navajas, Juan Carlos; Ye, Jiming

https://researchrepository.rmit.edu.au/esploro/outputs/9921858424301341/filesAndLinks?institution=61RMIT_INST\&index=null

Zeng, X., Zhou, X., Xu, J., Chan, S. M. H., Xue, C. C., Molero - Navajas, J. C., \& Ye, J. (2012). Screening for the efficacy on lipid accumulation in 3T3-L1 cells is an effective tool for the identification of new anti-diabetic compounds. Biochemical Pharmacology, 84(6), 830-837. https://doi.org/10.1016/j.bcp.2012.07.003 Document Version: Published Version

Published Version: https://doi.org/10.1016/j.bcp.2012.07.003

Repository homepage: https://researchrepository.rmit.edu.au (C) 2012 Elsevier Inc. All rights reserved.

Downloaded On 2023/04/26 22:50:53 +1000 


\title{
Screening for the efficacy on lipid accumulation in 3T3-L1 cells is an effective tool for the identification of new anti-diabetic compounds
}

\author{
Xiao-Yi Zeng ${ }^{\mathrm{a}, 1}$, Xiu Zhou ${ }^{\mathrm{a}, 1}$, Jun Xu ${ }^{\mathrm{b}}$, Stanley M.H. Chan ${ }^{\mathrm{a}}$, Charlie L. Xue ${ }^{\mathrm{a}}$, Juan C. Molero ${ }^{\mathrm{a}}$, Ji-Ming Ye ${ }^{\mathrm{a}, *}$ \\ ${ }^{a}$ Molecular Pharmacology for Diabetes Group, Health Innovations Research Institute and School of Health Sciences, RMIT University, Melbourne, Australia \\ ${ }^{\mathrm{b}}$ Center of Computer-Aided Drug Design, School of Pharmaceutical Sciences, Sun Yat-sen University, Guangzhou 510006, China
}

\section{A R T I C L E I N F O}

Article history:

Received 27 May 2012

Accepted 6 July 2012

Available online 20 July 2012

\section{Keywords:}

Drug discovery

Phenotypic screening

Lipid accumulation

Insulin resistance

Obesity

Fatty liver

\begin{abstract}
A B S T R A C T
Reducing lipid accumulation in insulin target tissues is critical for the treatment of type 2 diabetes. This study aimed to develop a biochemical assay in cells for high throughput (HTP) screening of anti-diabetic drugs by reducing lipid accumulation via different mechanisms. We designed a new method to extract triglyceride (TG) with $\mathrm{KOH}$ to allow biochemical quantification of TGs for HTP screening in 3T3-L1 cells. This new method was validated for its biochemical properties with identical results of TG obtained with or without $\mathrm{KOH}\left(r^{2}=0.9978, p<0.001\right)$ and a fourfold improvement in TG extraction recovery rate $(88-95 \%, p<0.001)$ as compared to the conventional chloroform/methanol extraction $(12-18 \%)$. The ability of this phenotype screening to capture potential anti-diabetic drugs was verified by pharmacological agents well known to alter lipid accumulation by different mechanisms including AMPK activators, fatty acid synthesis inhibitors, PPAR $\gamma$ activator and several lipogenic substrates. To further demonstrate the application of this screening tool for discovery of new anti-diabetic drugs, we screened $>200$ new candidates selected from Chinese medicine and identified 49 compounds from different classes which reduced TG content by $>50 \%$ at $1 \mu \mathrm{M}$ or $>75 \%$ at $10 \mu \mathrm{M}$. Finally, we tested two selected leads (albiflorin and oxymatrine) in vivo and confirmed their efficacy in reducing visceral adiposity, glucose intolerance and hepatic steatosis in high fat-fed or high fructose-fed mice. Our results indicate that screening for the efficacy on lipid accumulation in cells by biochemical quantification of TGs with $\mathrm{KOH}$ extraction is an effective tool for the identification of new anti-diabetic compounds.
\end{abstract}

(c) 2012 Elsevier Inc. All rights reserved.

\section{Introduction}

The prevalence of type 2 diabetes (T2D) is increasing at an alarming rate worldwide largely due to the increased prevalence of obesity. It has been estimated that there are around 200 million

Abbreviations: Abs, absorbance; ACC, acetyl-CoA carboxylase; AF, albiflorin; AMPK, AMP-activated protein kinase; ATG, arctigenin; BBR, berberine; BW, body weight; $\mathrm{CH}-\mathrm{Con}$, standard chow; C/M, chloroform/methanol; DMEM, Dulbecco's modified Eagles's medium; FA, fatty acid; FAS, fatty acid synthase; FBS, fetal bovine serum; FXR, farnesoid $\times$ receptor; GTT, glucose tolerance test; HFD, high fat diet; HTP, high throughput; IBMX, 3-isobutyl-1-methylxanthine; ip, intraperitoneal; $\mathrm{KOH}$, potassium hydroxide; $\mathrm{LDH}$, lactate dehydrogenase; OA, oleanolic acid; OM, oxymatrine; ORO, Oil Red O; PPAR, peroxisome proliferator-activating receptor; PSG, penicillinstreptomycin-glutamine; RSG, rosiglitazone; SREBP-1c, sterol regulatory elementbinding protein-1c; STD, standard; T2D, Type 2 diabetes; TCM, traditional Chinese medicine; TG, triglyceride; TOFA, 5-(tetradecyloxy)-2-furancarboxylic acid; TP, triterpenoid; Veh, vehicle.

* Corresponding author at: Molecular Pharmacology for Diabetes Group, Health Innovations Research Institute and School of Health Science, RMIT University, PO Box 71, Melbourne, Victoria 3083, Australia. Tel.: +61 39925 7419, fax: +61 399257178 .

E-mail address: jiming.ye@rmit.edu.au (J.-M. Ye).

${ }^{1}$ These authors contributed equally to the study (co-first authors). people suffering from T2D, and this figure is expected to increase to over 320 million by 2030 [1]. It has been established that insulin resistance (a reduced responsiveness of tissues, i.e. muscle and liver, to insulin) is a major factor linking obesity (particularly central adiposity) and T2D. At the pre-diabetic (insulin resistance alone) stage, blood glucose is maintained relatively normal via a compensatory increase in insulin secretion by the pancreas. However, prolonged insulin resistance may eventually result in beta-cell failure and the circulating insulin levels become insufficient to control blood glucose, leading to overt hyperglycaemia and the development of diabetes-associated complications.

Triglycerides (TGs) are commonly used as indicators of intracellular accumulation of lipids. Although TGs do not directly cause insulin resistance by themselves alone, they constitute the major storage of lipid intermediates that can interrupt insulin action by multiple mechanisms. For example, the intracellular forms of fatty acids (FAs) and long-chain fatty acyl-CoAs can inhibit hexokinase and glycogen synthase [2,3]; diacylglycerols can block insulin signalling pathways by promoting protein kinase C-dependent serine/threonine phosphorylation of IRS-1 [4]; furthermore, ceramide can dephosphorylate Akt and thus inhibiting its activity [5]. The toxicity of these lipid metabolites can also 
trigger other mechanisms including pro-inflammatory cytokines to exacerbate insulin resistance at a later stage [6]. Therefore, reducing ectopic lipid accumulation has been shown to reverse insulin resistance and obesity.

The cellular level of TG is determined by de novo lipogenesis (mainly by FA synthesis) and FA oxidation (mainly in mitochondria). One of the most attractive mechanisms to promote a reduction on intracellular lipid levels is the activation of AMPactivated protein kinase (AMPK). This protein phosphorylates and inhibits the activity of acetyl-CoA carboxylase (ACC). ACC mediates the first step in the de novo synthesis of fatty acids and simultaneously, its product malonyl-CoA blocks the entry of FAs in the mitochondria for oxidation by inhibiting the carnitine palmitoyltransferase shuttle system. Thus, AMPK has been an attractive target for the discovery of drugs with potential efficacy for T2D and obesity [7]. The other mechanism to reduce cellular TG level is the inhibition of FA synthesis, particularly in adipose tissue and liver, because these tissues exhibit elevated de novo lipogenesis capacity that can exacerbate lipid accumulation. Indeed, a number of compounds acting at key steps of the lipogenic pathway have been shown to reduce obesity, hepatic steatosis and/or insulin resistance, such as specific inhibitors of fatty acid synthase, C75 and cerulenin [8], ACC inhibitor 5-(tetradecyloxy)-2-furancarboxylic acid (TOFA) [9] or sterol regulatory element-binding protein-1c (SREBP-1c) [10].

Here, we established a HTP phenotypic screening assay with a cellular TG level as a measurement capable of capturing the lipidlowering efficacy from actions at different sites in lipid metabolic pathways. This newly developed screening assay was made possible by using $\mathrm{KOH}$ based on its ability to lyse cells and hydrolyse TGs [11] to overcome the obstacles encountered with the conventional organic solvent. Following the validation by a series biochemical and pharmacological tests, we used this HTP assay to screen more than 200 candidates selected from different classes of compounds from traditional Chinese medicine (TCM). Our results identified over 40 potential anti-diabetic compounds and among them we confirmed the efficacy of newly identified compounds albiflorin in high fat-fed mice and oxymatrine in high fructose-fed mice. These findings provide strong support for the application of this screening assay for the discovery of novel antiobese and anti-diabetic drugs.

\section{Materials and methods}

\subsection{Regents, chemicals and test compounds}

Insulin, dexamethasone, DMSO, biotin, 3-isobutylxanthine (IBMX), AICAR, berberine (BBR), oleanolic acid (OA), arctigenin (ATG), betulin, C75, cerulenin, 5-(tetradecyloxy)-2-furancarboxylic acid (TOFA), rosiglitazone (RSG), fructose, genipin $(\geq 98.0 \%)$, bile acids including cholic acid, deoxycholic acid and chenodeoxycholic acid, as well as chemicals and solvents used in various buffers were purchased from Sigma-Aldrich (Melbourne, Australia). Tissue culture reagents were obtained from Invitrogen (Melbourne, Australia). The compounds tested were kindly supplied via or by Dr Lihong Hu and Dr Yang Ye of the Shanghai Institute of Materia Medica, Chinese Academy of Sciences (Shanghai, China) and Dr Jun Xu of the School of Pharmaceutical Sciences, Sun Yat-sen University (Guangzhou, China). Specifically, albiflorin (98.0\%) was obtained from Baoding Yunxin Trade (Hebei, China) and oxymatrine (98.0\%) from Hangzhou Tianlong Biotechnology (Hangzhou, China). Tiliroside $(>98.0 \%$ ) was obtained from Meryer Chemical Technology (Shanghai, China). The derivatives of albiflorin, oxymatrine, berberine, genipins and tiliroside were developed at Shanghai Institute of Materia Medica by modifying their chemical structures. Triterpenoids were obtained from the purification of the extracts of bitter melon as we recently described [12]. FXR ligands and other bile acid analogues were developed in Sun Yat-sen University. Other test compounds were obtained from the chemical library of Sun Yat-sen University after computerbased virtue screening against the targets for lipid metabolism [13]. To improve the likelihood of success and minimize the numbers for screening, we selected the candidates with a wide range of chemical diversity by one of the two following virtual screening processes [12-14], The first virtual screening was based on careful evaluation of documented (or reported) effects implicated for diabetes or related conditions as used in our recent identification of BBR and its derivatives from Golden Seal [14] and cucurbitane triterpenoids from Bitter Melon [12]. The second process involved computer modelling possible binding of test compounds to cellular targets, such as the farnesoid $\mathrm{X}$ receptor (FXR) (a regulator of lipid metabolism [15]), in the software available to us by the similar approach as described for the liver $\mathrm{X}$ receptor [13].

\subsection{Cell culture}

3T3-L1 mouse embryonic fibroblasts (American Type Culture Collection, Manassas, VA) were cultured in Dulbecco's modified Eagles's medium (DMEM) supplemented with $10 \%(\mathrm{v} / \mathrm{v})$ foetal bovine serum (FBS) and $1 \%(\mathrm{v} / \mathrm{v})$ penicillin-streptomycin-glutamine (PSG) at $37{ }^{\circ} \mathrm{C}$ in $5 \% \mathrm{CO}_{2}$. The 3T3-L1 pre-adipocytes were seeded into 48- or 96-well micro-plates and allowed to reach full confluence. To induce pre-adipocyte differentiation, 2-day postconfluent cells were incubated in differentiation medium (DMEM containing $10 \% \mathrm{FBS}, 1 \% \mathrm{PSG}, 2 \mu \mathrm{g} / \mathrm{ml}$ insulin, $100 \mathrm{ng} / \mathrm{ml}$ dexamethasone, $500 \mu \mathrm{M}$ IBMX and $10 \mathrm{ng} / \mathrm{ml}$ biotin) for 3 days before switching to post-differentiation medium (DMEM with 10\% FBS, $1 \%$ PSG and $2 \mu \mathrm{g} / \mathrm{ml}$ insulin) for additional 3 days. To assess their effects on lipid accumulation, the test compounds were dissolved in DMSO and supplemented at the concentrations indicated in the figure legends throughout the course of the differentiation. Test compounds were made as stocking solutions and stored at $-20^{\circ} \mathrm{C}$ in aliquots. They were added on day 0 in differentiation medium (for 3 days) and replaced with another aliquot in post-differentiation medium on day 3 (for another 3 days). 3T3-L1 cells cultured in DMEM supplemented with $0.1 \%$ DMSO were used as a vehicle control for all experiments. Cells were monitored and photographed using the Nikon TS100F inverted microscope and camera (Nikon Instruments Inc., USA).

\subsection{Design for a novel technique of TG extraction for biochemical assay}

At the end of the 3-day post-differentiation medium incubation, the culture medium was removed and the cells were washed twice with ice-cold PBS buffer $\left(0.2 \mathrm{M} \mathrm{NaCl}, 10 \mathrm{mM} \mathrm{Na}_{2} \mathrm{HPO}_{4}, 3 \mathrm{mM} \mathrm{KCl}\right.$, $2 \mathrm{mM} \mathrm{KH} \mathrm{PO}_{4}, \mathrm{pH} 7.4$ ), before subjected to drying in a $37^{\circ} \mathrm{C}$ incubator (Ratek Instruments, Australia). Cells were lysed and TGs were extracted by the addition of $\mathrm{KOH}$ (final concentration $50 \mathrm{mM}$ ) followed by incubation at $60{ }^{\circ} \mathrm{C}$ for $10 \mathrm{~min}$ and centirfuged at $4000 \mathrm{rpm}$ for $5 \mathrm{~min}$. For the conventional extraction method [16], cells were scraped immediately following the addition of the chloroform/methanol (2:1) solution. The cell lysate was mixed with $0.6 \% \mathrm{NaCl}$ solution and centrifuged at $2000 \mathrm{rpm}$ for $10 \mathrm{~min}$. The bottom organic layer containing the TG was collected and dried under nitrogen gas at $45^{\circ} \mathrm{C}$. TG was reconstituted in ethanol for the measurement using a commercial Peridochrom TG GPOPAP kit (Roche Diagnostics, Castle Hill, New South Wales, Australia) following the manufacturer's instructions in a spectrophotometer. To evaluate the influence of $\mathrm{KOH}$ on the enzymatic assay, the TG kit was tested in the presence of $50 \mathrm{mM} \mathrm{KOH}$ (used 
for TG extraction) at various concentrations of intralipid (SigmaAldrich, Melbourne, Australia) as a TG donor [3,4]. The recovery rate of TG extraction from 3T3-L1 cells was assessed by adding known amounts of TGs from the intralipid before the extraction either by $\mathrm{KOH}$ or the $\mathrm{C} / \mathrm{M}$ mixture.

\subsection{Oil red $O(O R O)$ staining}

Oil red O (ORO) staining was used in combination with light microscopy to assess pre-adipocyte differentiation as previously described [17]. Briefly, the treated differentiated 3T3-L1 cells were washed twice with ice-cold PBS buffer and fixed in 10\% neutral formalin overnight before staining with a $0.35 \%$ (w/v) ORO solution in isopropanol for $10 \mathrm{~min}$. Excess stains were then removed by rinsing the cells under water and then dried before microscopic examination. For quantitative analysis, ORO was eluted with isopropanol and the absorbance was measured at $485 \mathrm{~nm}$ in a spectrophotometer.

\subsection{Determination of lactate dehydrogenase $(L D H)$ release}

After cells were treated with compounds for 6 days, the medium and lysate were collected to measure the activity of lactate dehydrogenase (LDH) released into the culture medium from cells. The medium was collected and centrifuged at $1000 \mathrm{rpm}$ at $4{ }^{\circ} \mathrm{C}$ for $10 \mathrm{~min}$ to remove detached cells. To measure the intracellular LDH activity, 3T3-L1 cells were lysed by two freezethaw cycles (chilling the plate at $-80^{\circ} \mathrm{C}$ for approximately $30 \mathrm{~min}$ followed by thawing at $37^{\circ} \mathrm{C}$ for $15 \mathrm{~min}$ ) after wash with ice-cold PBS buffer. The cell lysate was then centrifuged at $1000 \mathrm{rpm}$ for $4 \mathrm{~min}$, and the supernatant was stored at $4{ }^{\circ} \mathrm{C}$ until ready to use. The enzyme activity in the whole cell lysate and medium was determined by the CytoTox $96^{\circledR}$ Non-Radioactive Cytotoxicity Assay kit (Promega, Melbourne, Australia) according to the manufacturer's protocol. Briefly, $50 \mu \mathrm{l}$ of the diluted medium $(1: 5)$ or cell lysate $(1: 10)$ were transferred onto a plate followed by addition of the reconstituted substrate mixture. The absorbance of the samples was read at $490 \mathrm{~nm}$ using the Polarstar Optima microplate reader (BMG Labtech, Germany). LDH released into the medium was expressed as a percentage of the total LDH activity (ratio of LDH in lysate and in the medium) as described.

\subsection{Animal studies}

Male C57BL/6J mice (10 weeks old) were purchased from the Animal Resources Centre (Perth, Australia). The animals were kept in a temperature-controlled room $\left(22 \pm 1^{\circ} \mathrm{C}\right)$ on a 12-h light/dark cycle with free access to food and water. Animals were randomly assigned to receive a high fat (60\%) diet (HFD) ad libitum to generate insulin resistance $[12,14]$ or a high fructose (35\% fructose and $35 \%$ starch) diet (HFru) to generate hepatic steatosis [18]. Animals were fed for 8 weeks with or without albiflorin (AF, $100 \mathrm{mg} /(\mathrm{kg}$ day) in HFD), for the final 2 weeks. In a separate study, HFru-fed mice received oxymatrine (OM, $100 \mathrm{mg} /(\mathrm{kg}$ day) in diet, 1 week) after 3-day HFrufeeding (unless indicated otherwise). Body weight, food intake and fasting blood glucose level were monitored on weekly basis. After two weeks of AF treatment in HFD mice, glucose tolerance test (glucose load $2 \mathrm{~g} / \mathrm{kg}$ BW, i.p.) was performed after 6-h fasting. Glucose level of blood collected from the tail vein was measured at designed time points using the Accu-Chek glucometer (Roche Diagnostics, Castle Hill, New South Wales, Australia). The effect of OM on glucose tolerance in HFru-fed mice was assessed after 2 weeks of treatment at a glucose load of $3 \mathrm{~g} / \mathrm{kg}$ (i.p.) based on our previous studies [18]. All animal experiments were approved by the Animal Ethics Committee of RMIT University (Approval No \#1012) following the guidelines issued by the National Health and Medical Research Council of Australia.

\subsection{Statistical analyses}

Data were presented as means $\pm \mathrm{SE}$. Unpaired student test was performed for comparison of relevant groups. Pearson's two-sided correlation was used for correlation calculation. Differences at $p<0.05$ were considered to be statistically significant.

\section{Results}

\subsection{Validation of the novel TG extraction method for HTP biochemical assay}

The conventional method for the extraction of TGs from tissues with organic solvents requires a series of steps including the separation of organic and aqueous phases [16]. Thus we designed a one-step method to extract TG with the use of $\mathrm{KOH}$ based on its ability to lyse cells and hydrolyse TGs [11] (Fig. 1). We first tested whether or not $\mathrm{KOH}$ may influence the biochemical assay. Fig. 2A shows that at the concentration $(50 \mathrm{mM})$ designed for TG extraction $\mathrm{KOH}$ did not have any significant influence on the biochemical assay for the quantification of TG. The obtained results in the presence of $\mathrm{KOH}$ were almost identical to those obtained in the absence of $\mathrm{KOH}$ as indicated by a high degree of linear correlation $\left(Y=1.1416 X, r^{2}=0.9978, p<0.001\right)$. We next examined if $\mathrm{KOH}$ could extract a similar amount of TGs from cells, by adding 1.0 or $2.5 \mu \mathrm{g} \mathrm{TG}$ (50-100\% of TG from 3T3-L1 adipocytes) to a cell culture plate. As shown in Fig. 2B, the recovery rates of TGs extracted with $\mathrm{KOH}$ at these two concentrations of added TG were $87.8 \pm 4.6 \%$ and $95.1 \pm 0.5 \%$, respectively. These recovery rates were approximately four times of those extracted by $\mathrm{C} / \mathrm{M}(12.1 \pm 8.9$ and $18.0 \% \pm 8.4 \%$, respectively, $p<0.001)$. Furthermore, the obtained values of TGs extracted with $\mathrm{KOH}$ were quantitative in proportion to amount added $(0.88 \pm 0.05 \mu \mathrm{g}$ from $1.0 \mu \mathrm{g}$ and $2.4 \pm 0.01 \mu \mathrm{g}$ from $2.5 \mu \mathrm{g}$ ) (Fig. 2C).

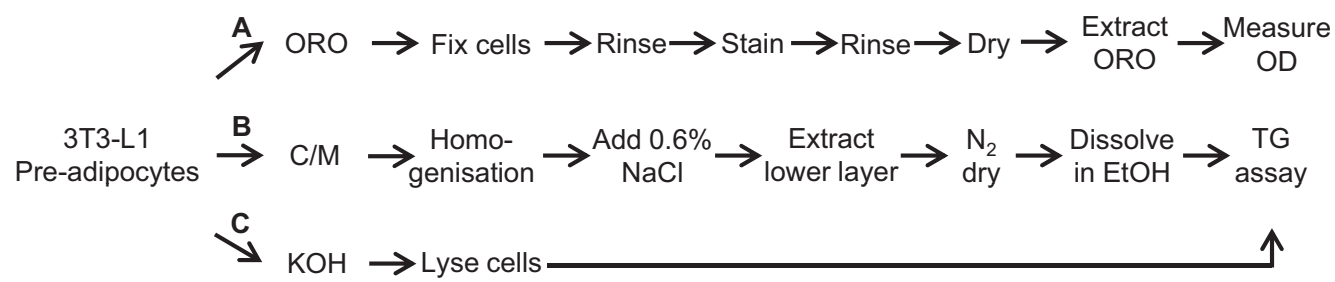

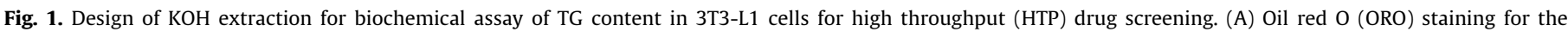

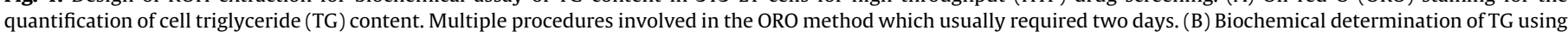

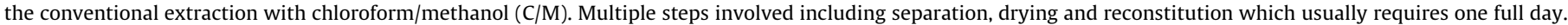

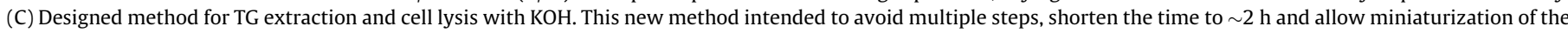
biochemical assay of TG for HTP drug screening. 
A

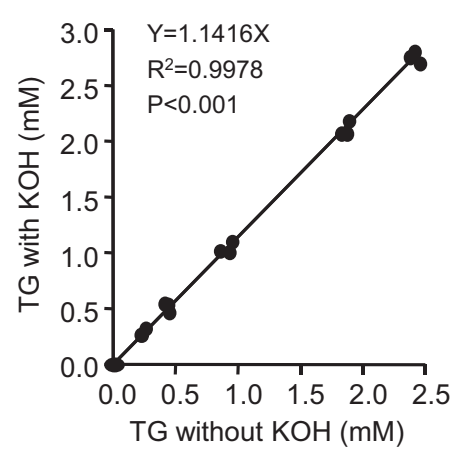

B

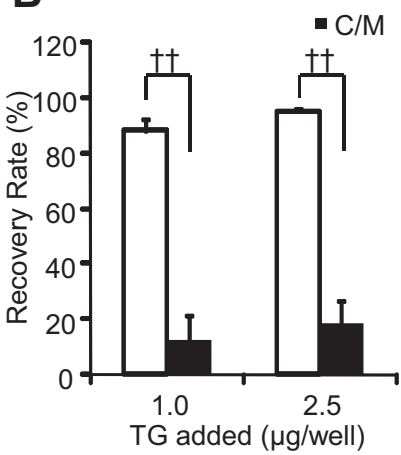

C

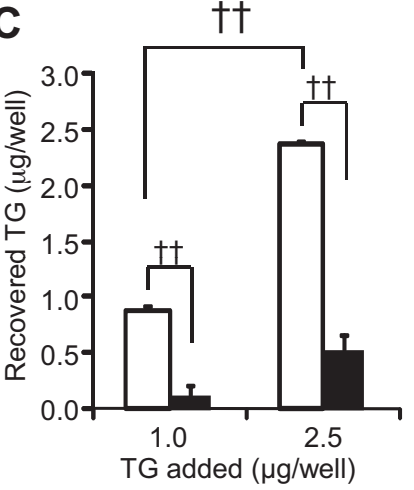

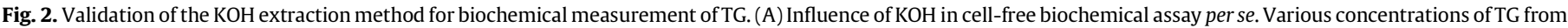

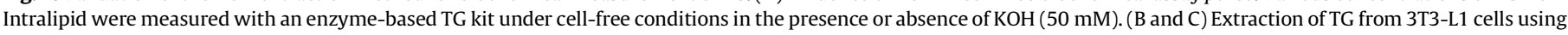

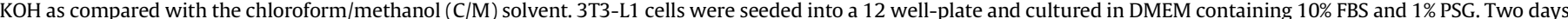

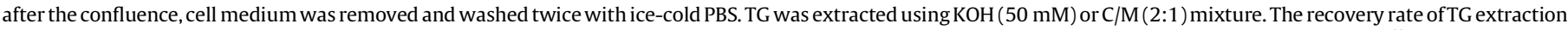
was calculated from values obtained with or without added TG (1.0 or $2.5 \mu \mathrm{g}$ from Intralipid). Data are means $\pm \mathrm{SE}$ from three independent experiments. ${ }^{\dagger \dagger} p<0.01$.

\subsection{Validation of the detectability of TG altered by known pharmacologic agents}

In order to verify the ability of this new assay as an end-point measurement to capture the effect on lipid accumulation induced
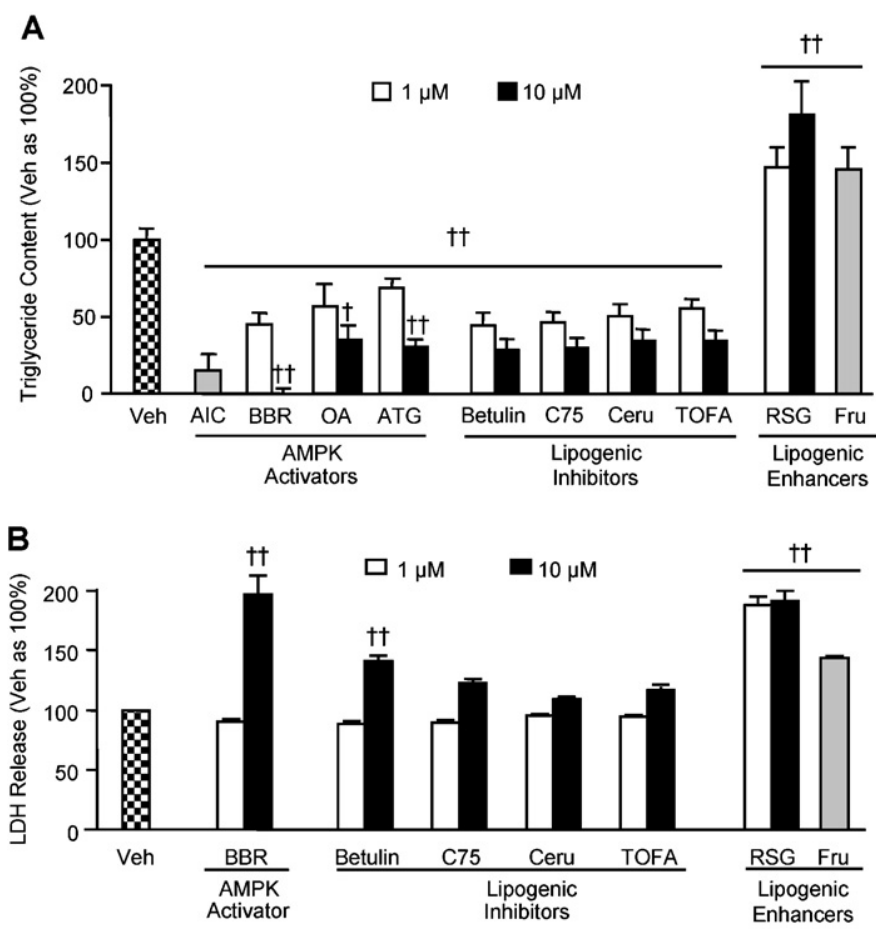

Fig. 3. Validation of the TG assay as an endpoint screening for drugs acting at different sites of lipid metabolism. (A) Assessment of the ability of the new screening assay to detect intracellular lipid accumulation. 3T3-L1 cells were seeded into a 48 well-plate and cultured in DMEM. Two days after confluence, cells were incubated with differentiation medium (DMEM containing 10\% FBS, 1\% PSG, $2 \mu \mathrm{g}$ / $\mathrm{ml}$ insulin, $100 \mathrm{ng} / \mathrm{ml}$ dexamethasone, $500 \mu \mathrm{M}$ IBMX and $10 \mathrm{ng} / \mathrm{ml}$ biotin) for 3 days. Differentiated cells were cultured for additional 3 days in DMEM with $2 \mu \mathrm{g} / \mathrm{m}$ insulin (post-differentiation medium) with modulators affecting lipogenesis and/or lipolysis. (B) Lactate dehydrogenase (LDH) release. The cell medium and lysate were collected 6 days after the incubation with the modulators. LDH release was determined by its activity in the medium relative to the cell lysate and corrected with the vehicle (Veh) control. These lipogenesis/lipolysis modulators included (i) AMPK activators - AICAR (AIC, $500 \mu \mathrm{M}$ ), berberine (BBR), oleanolic acid (OA) and arctigenin (ATG); (ii) lipogenic inhibitors - betulin (SREBP-1c inhibitor), C75 (FAS inhibitor), cerulenin (Ceru, FAS inhibitor), TOFA (ACC inhibitor); and (iii) lipogenic enhancers - rosiglitazone (RSG, PPAR $\gamma$ agonist) and fructose (Fru, $20 \mathrm{mM}$ ). Data are means \pm SE from three independent experiments. ${ }^{\dagger \dagger} p<0.01$ vs Veh. by different upstream mechanisms, we tested a number of pharmacological agents well known to promote FA oxidation, inhibit or enhance lipogenesis in our newly developed assay system. As expected, the AMPK activators AICAR [19], BBR [14], oleanolic acid (OA) [12], arctigenin (ATG) [20] all reduced the TG levels significantly $(p<0.01)$ at 1 and/or $10 \mu \mathrm{M}$ (Fig. 3A). Detailed examination revealed a dose-depend efficacy for these agents
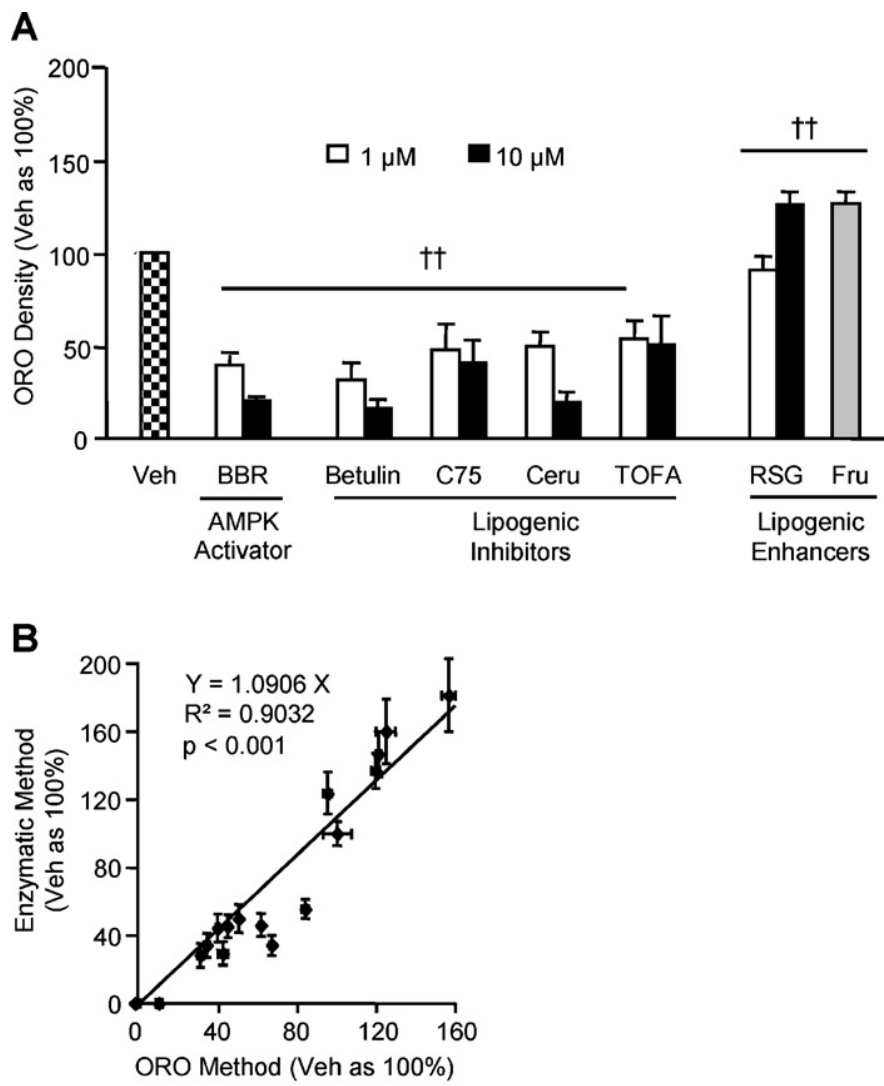

Fig. 4. Comparison of results obtained from the method of ORO staining. 3T3-L1 cells were cultured and incubated under the same conditions as described in the legend of Fig. 3. At the end of the experiment, cells were stained with ORO for the quantification of TG content. (A) Quantified results by measuring the optical density of ORO stained cells after the incubation with lipogenesis/lipolysis modulators. (B) Correlation of the results from the new assay using the new $\mathrm{KOH}$-enzyme method $v s$ the ORO staining. Data are means \pm SE from three independent experiments. ${ }^{\dagger \dagger} p<0.01$ vs Veh. 
(Fig. S1). Similar results were observed after the incubation with C75 and cerulenin (specific FAS inhibitors [9]), betulin (specific SREBP-1c inhibitor [10]), and tetradecyloxyfuroic acid (TOFA, specific ACC inhibitor [9]) (all $p<0.05$, Fig. 3A). In contrast, incubation with $20 \mathrm{mM}$ fructose (Fru, lipogenic substrate [21]) or 1-10 $\mu \mathrm{M}$ rosiglitazone (RSG, specific PPAR $\gamma$ agonist [22]) increased TG accumulation by more than $150 \%$ ( $p<0.05$ for both).

To exclude a possibility of the decrease in TG content resulting from cytotoxicity, we determined the LDH activity in the cultured media as an indicator of cell damage. Fig. 3B shows that apart from the higher concentration $(10 \mu \mathrm{M})$ of BBR and betulin which moderately elevated the LDH activity to the similar levels of Fru and RSG, there was no significant increase in LDH activity for other test agents at the concentrations which reduced TG content. Consistent with the results of LDH, the cells displayed a healthy morphology in response to these treatments (Fig. S2).

\subsection{Comparison with results obtained from ORO staining}

As ORO staining is widely used to screen the changes in lipid content in cultured cells [17], we compared the values of intracellular lipid content obtained from new method with the semi-quantitative ORO method. As shown in Fig. 4A, the ORO staining method confirmed all the responses we detected using our new screening assay. The obtained values of TG were all significantly reduced by lipogenesis inhibitors BBR, betulin, C75, cerulenin and TOFA ( $>40 \%$ ) but increased by Fru and RSG ( $>130 \%)$ under the experimental conditions (all $p<0.01$ ). The mean values of results obtained from these two assays were significantly correlated $\left(Y=1.0906 X, r^{2}=0.9032, p<0.001\right)$ (Fig. 4B).

\subsection{Screening of test compounds for their effects on TG accumulation} in 3T3-L1 adipocytes

Following the biochemical and pharmacological validations as described above, we applied this new screening protocol in 48-well plates (as illustrated in Fig. 5A) in an attempt to identify potential lipid-lowering drugs by targeting the compounds developed from traditional Chinese medicine (TCM). Among the 207 candidates selected for the screening, we detected 49 hits (compounds with efficacy in reducing TG accumulation by $>50 \%$ at $1 \mu \mathrm{M}$ or $>75 \%$ at $10 \mu \mathrm{M}$ ) (Fig. 5B). These identified hits included several classes of compounds with different core structures, particularly derivatives of BBR (Fig. 6A), triterpenoids (TP, Fig. 6B), tiliroside (Fig. 6C), oxymatrine (Fig. 6D), albiflorin (Fig. 6E) and ligands for farnesoid $\mathrm{X}$ receptor (FXR, Fig. 6F).

\subsection{Demonstration of therapeutic effects in vivo of leads selected from the identified hits}

We next tested if our new screening system may guide the identification of new leads with anti-diabetic and/or anti-steatotic efficacy in vivo by confirming the therapeutic effect of the elected candidates in a well-recognized model of insulin resistance and obesity (HFD), and hepatic steatosis (HFru), respectively. Based on the screening results (illustrated in Fig. 5), we elected to examine albiflorin (AF, an analogue of paeoniflorin). AF has been suggested to be a key ingredient of Paeonia radix which has been shown to exert acute effect in reducing blood glucose in diabetic mice [23]. As shown in Table 1, HFD mice with oral administration of AF at $100 \mathrm{mg} /(\mathrm{kg}$ day) (based on the reported i.p. dose of $30 \mathrm{mg} / \mathrm{kg}$ used

A
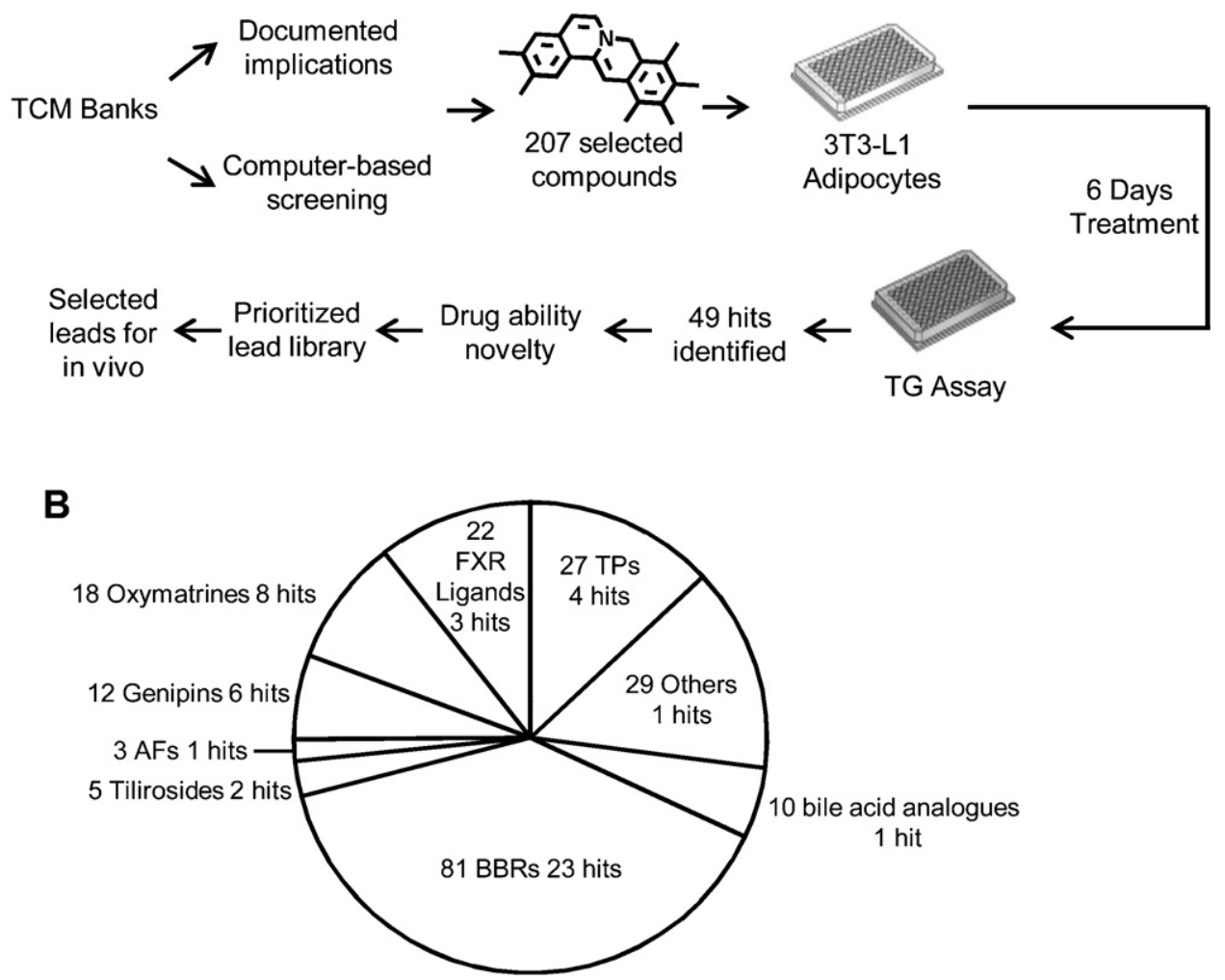

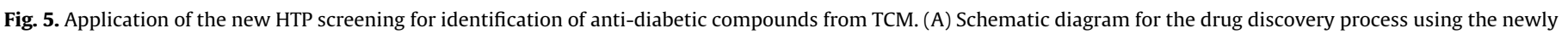

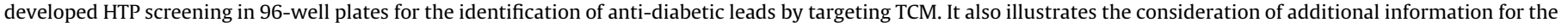

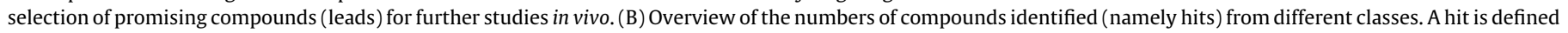
as a compound with the efficacy in lowering TG for $>50 \%$ at $1 \mu \mathrm{M}$ or $>75 \%$ at $10 \mu \mathrm{M}$. Results were obtained from three independent experiments. 
A

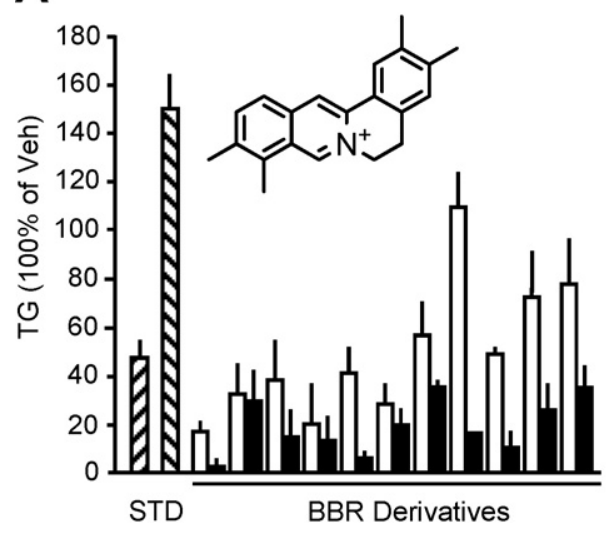

B

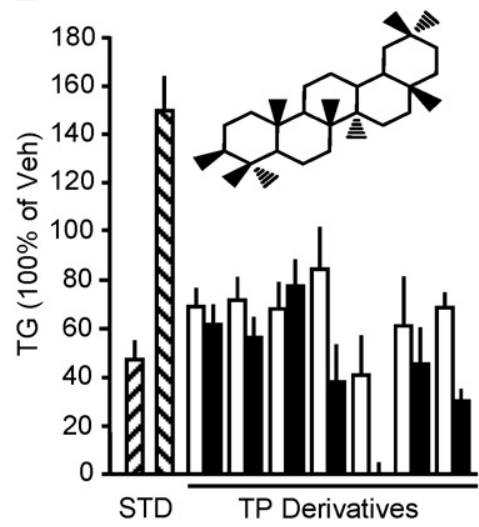

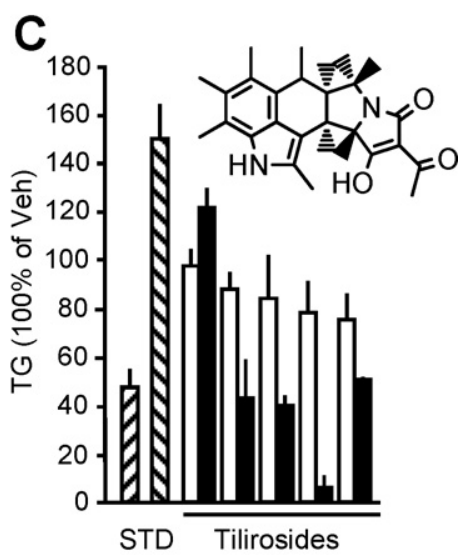
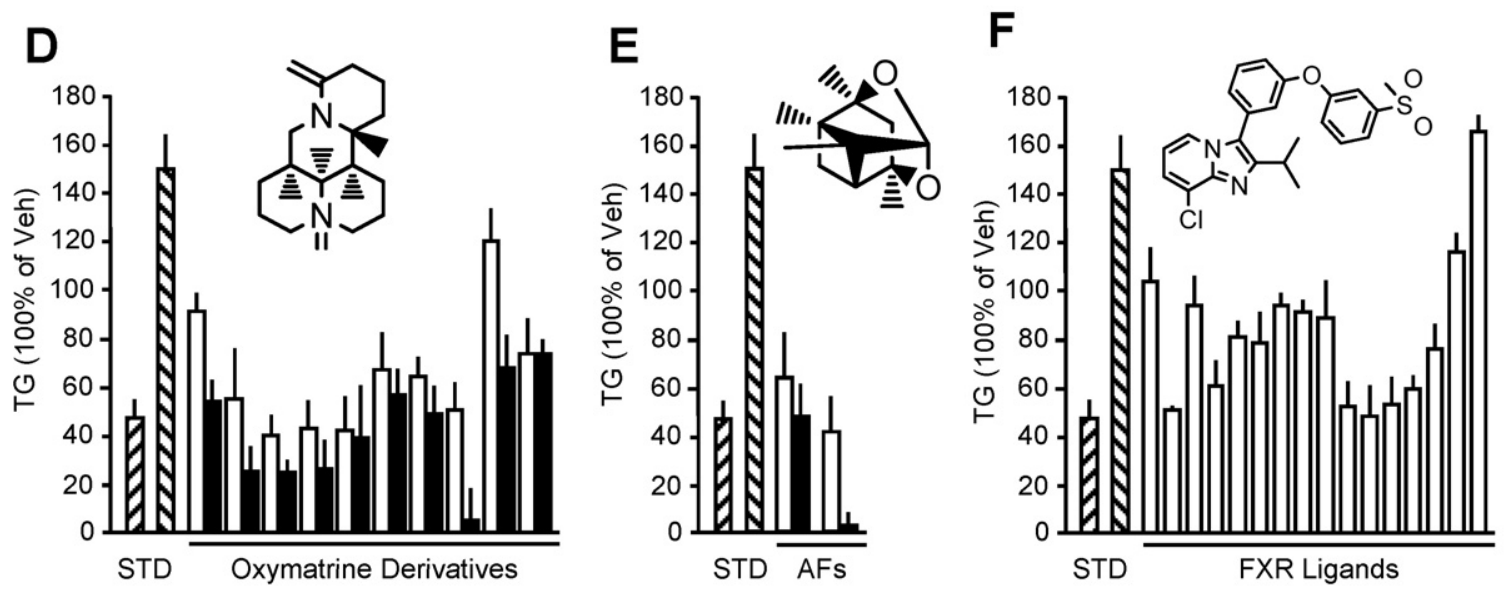

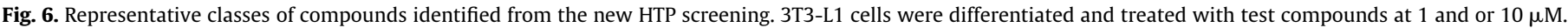

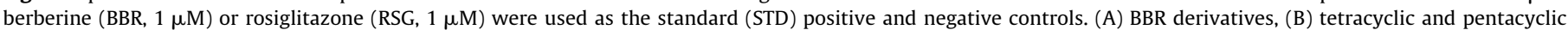

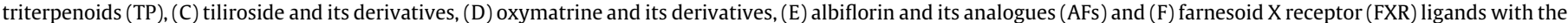
core structure for each class. Data are means \pm SE from one independent experiment and for each of experiment triplicates were performed.

in mice [23]) for 2 weeks had significantly lesser gain of body weight (by $45 \%, p<0.01$ ) and visceral adiposity (indicated by a $68 \%$ reduction in epididymal fat mass, $p<0.01$ ) compared with control HFD mice. These effects occurred in the absence of any influence on caloric intake $(p>0.05)$. Consistent with the efficacy on obesity, AF treatment significantly improved glucose tolerance, as indicated by $43 \%$ reduction $(p<0.01)$ in the area under the curve (iAUC) for

Table 1

Metabolic effects of the identified lead albiflorin in insulin resistant mice induced by chronic high fat feeding.

\begin{tabular}{llll}
\hline & CH-Con & HFD-Con & HFD-AF \\
\hline Body weight prior treatment $(\mathrm{g})$ & $26.4 \pm 0.5$ & $27.6 \pm 0.6$ & $27.0 \pm 0.4$ \\
Body weight after treatment $(\mathrm{g})$ & $27.2 \pm 0.5$ & $28.8 \pm 0.8^{*}$ & $27.5 \pm 0.4$ \\
Body weight gain $(\mathrm{g})$ & $0.81 \pm 0.08$ & $1.20 \pm 0.17^{*}$ & $0.66 \pm 0.14^{\dagger \dagger}$ \\
Caloric intake $(\mathrm{kcal} /($ mouse day)) & $10.6 \pm 0.5$ & $10.2 \pm 0.2$ & $11.4 \pm 0.7$ \\
Epididymal fat mass (\% BW) & $1.24 \pm 0.12$ & $3.32 \pm 0.25^{* *}$ & $1.06 \pm 0.08^{\dagger \dagger}$ \\
Blood glucose $(\mathrm{mM})$ & $9.4 \pm 0.3$ & $10.8 \pm 0.3$ & $11.8 \pm 0.3$ \\
ipGTT iAUC $(\mathrm{mM} \times 90 \mathrm{~min})$ & $740 \pm 70$ & $1270 \pm 83^{* *}$ & $722 \pm 64^{\dagger \dagger}$ \\
\hline
\end{tabular}

Body weight gain, daily food intake, fasting glucose, and epididymal fat pad weights for $\mathrm{C} 57 \mathrm{BL} / 6 \mathrm{~J}$ mice fed 8 weeks on either standard chow ( $\mathrm{CH}-\mathrm{Con}$ ), high fat diet (HFD-Con), or HFD supplemented with albiflorin (AF, $100 \mathrm{mg} /(\mathrm{kg}$ day) for 2 weeks) (HFD-AF). Glucose tolerance test was conducted with an intraperitoneal injection (ipGTT) of glucose load of $2 \mathrm{~g} / \mathrm{kg}$. iAUC, incremental area under the curve of blood glucose during the ipGTT. Data represents the mean \pm SE of 7-10 mice for each group.

${ }^{*} p<0.05$ vs $\mathrm{CH}-\mathrm{Con}$.

** $p<0.01$ vs $\mathrm{CH}-\mathrm{Con}$.

i† $p<0.01$ vs HFD-Con. blood glucose during the ipGTT. To further test the application of new screening system, we examined the effect of oxymatrine (OM) because this alkaloid (rich in Sophora flavescens) has been used in humans as a safe oral drug in treatment of hepatic fibrosis [24]. As shown in Table 2, oral administration of OM for one week was able

Table 2

Metabolic effects of the identified lead oxymatrine on hepatic steatosis induced by high fructose feeding.

\begin{tabular}{lrrc}
\hline & \multicolumn{1}{c}{ CH-Con } & \multicolumn{1}{c}{ HFru-Con } & \multicolumn{1}{c}{ HFru-OM } \\
\hline Body weight prior treatment $(\mathrm{g})$ & $25.8 \pm 0.6$ & $25.8 \pm 0.7$ & $25.3 \pm 0.6$ \\
Body weight after treatment $(\mathrm{g})$ & $26.3 \pm 0.6$ & $26.4 \pm 0.6$ & $25.2 \pm 0.6$ \\
Body weight gain $(\mathrm{g})$ & $0.5 \pm 0.2$ & $0.6 \pm 0.1$ & $0.0 \pm 0.1^{*}{ }^{*} \dagger$ \\
Caloric intake $(\mathrm{kcal} /(\mathrm{mouse}$ day) & $11.3 \pm 1.1$ & $14.3 \pm 0.6$ & $14.4 \pm 1.2^{* *}$ \\
Epididymal fat mass $(\% \mathrm{BW})$ & $1.1 \pm 0.0$ & $1.7 \pm 0.1^{* *}$ & $1.5 \pm 0.1^{* *}$ \\
Liver triglyceride $(\mu \mathrm{mol} / \mathrm{g})$ & $11.6 \pm 1.4$ & $23.9 \pm 2.5^{* *}$ & $13.5 \pm 1.2^{\dagger \dagger}$ \\
Blood glucose $(\mathrm{mM})$ & $9.8 \pm 0.3$ & $11.7 \pm 0.6^{*}$ & $9.8 \pm 0.6$ \\
ipGTT $^{\mathrm{a}}$ iAUC $(\mathrm{mM} \times 90 \mathrm{~min})$ & $981 \pm 90$ & $1256 \pm 60^{*}$ & $1050 \pm 92^{\dagger}$ \\
\hline
\end{tabular}

Body weight gain, daily food intake, epididymal fat pad weights and liver triglyceride content for $\mathrm{C} 57 \mathrm{BL} / 6 \mathrm{~J}$ mice fed 1 week on either standard chow $(\mathrm{CH}-$ Con), high fructose diet (HFru-Con), or HFru supplemented with oxymatrine (OM, $100 \mathrm{mg} /(\mathrm{kg}$ day $))$ (HFru-OM).

a ipGTT was performed after two weeks of oral administration of OM at a glucose load of $3 \mathrm{~g} / \mathrm{kg}$ BW. Data represents the mean $\pm \mathrm{SE}$ of 7-13 mice for each group. ${ }^{*} p<0.05$ vs $\mathrm{CH}-\mathrm{Con}$.

$p<0.01$ vs $\mathrm{CH}-\mathrm{Con}$

$\dagger p<0.05$ vs HFru-Con.

i† $p<0.01$ vs HFru-Con. 
to reduce body weight gain $(p<0.05)$ and eliminating hepatic steatosis $(p<0.05)$ in HFru fed mice, a model of fatty liver due induced by de novo lipogenesis [18]. After 2 weeks of administration of OM, glucose tolerance was significantly improved (iAUC: $1050 \pm 92$ vs $1260 \pm 60 \mathrm{mM} \times 90 \mathrm{~min}, p<0.05, n=8 /$ group $)$.

\section{Discussion}

As excess accumulation of lipids in cells is a hallmark of obesity and insulin resistance [25], elimination of elevated cellular lipid levels is an effective approach for the treatment of these pathological conditions [2-4]. Based on this concept, we established a biochemical assay of TGs in cells as an HTP screening tool by designing a new lipid extraction method in order to identify compounds with potential efficacy for obesity and associated metabolic diseases. With this newly developed screening tool, we screened 207 compounds selected from two TCM banks based on extensive evaluation of TCM database $[12,14]$ and/or computer modelling $[13,26]$. Among them, 49 showed an efficacy in reducing TG by $>50 \%$ at $1 \mu \mathrm{M}$ or $>75 \%$ at $10 \mu \mathrm{M}$, which we regarded as identified hits. Based on their drug-like properties and sufficient quantity available to us, we demonstrated the in vivo anti-diabetic and anti-steatotic effects of two novel leads (AF and OM) identified from these hits. Our results provide the proof of concept for the use of this HTP biochemical screening tool for the discovery of novel anti-diabetic drugs.

Up till now, the ORO staining has been commonly used in adipocytes to screen the efficacy on lipid content [17], particular for drugs promoting cell differentiation such as PPAR $\gamma$ agonists [22]. However, this method involves multiple processes which are time consuming and can be labour intensive when used at a large scale. Furthermore, there is concern about the specificity and the inaccuracy of the results obtained from the ORO staining [17]. Therefore, it would be advantageous to develop an HTP screening for the cell TG levels based on a biochemical assay of TG widely used for in plasma and tissue samples. To overcome the technical obstacles in applying the biochemical assay for HTP screening, we designed a method of TG extraction using $\mathrm{KOH}$ based on its ability to lyse cells and hydrolyse TGs [11]. This new method provides advantages over the organic extraction that requires multiple steps, allowing the biochemical assay performed in a micro-plate for HTP drug screening. Importantly, $\mathrm{KOH}$ extraction produced an almost full recovery of TG (88-95\%), which is far more superior to the $12-18 \%$ of recovery obtained by the $\mathrm{C} / \mathrm{M}$ method. Our new procedure also substantially improves the efficiency of whole TG assay (approximately $2 \mathrm{~h}$ ) compared to the ORO staining and the $\mathrm{C} /$ $\mathrm{M}$ extraction method, which usually require much longer time.

Cellular TG level can be regulated by either the lipogenic and FA oxidative pathways. The HTP assay established with the new extract method was able to capture the expected effects of welldefined pharmacological agents in lipid metabolism. Among them, AICAR, BBR, bitter melon triterpenoid and ATG have been demonstrated to activate AMPK in previous studies including ours $[12,14,19,20]$. Activated AMPK can reduce cellular lipid accumulation by promoting FA oxidation [27] and inhibiting lipogenesis [28]. Decreased TG was also evident, as predicted, in the presence of lipogenic inhibitors acting at the site of SREBP-1c (betulin, [10]), ACC (TOFA [9]) or FAS (C75 and cerulenin [9]). Furthermore, our results also showed that our screening system was sensitive in detecting increased TG accumulation in response to lipogenic substrates (indicated by fructose [21]) and PPAR $\gamma$ agonists (indicated by RSG [22]). These results verify the suitability of this HTP screening to capture potential anti-diabetic drugs which alter lipid metabolisms by acting at different cellular sites.

Recently, we identified two anti-diabetic compounds such as BBR and cucurbitane triterpenoids from the plants used in TCM for the treatment of diabetes and related conditions [12,14]. Compared with a random screening of natural products, TCM has been a much more fruitful resource as it enables us to perform careful prior analysis [12,14], as well as computation [13] to reduce the large numbers of compounds to a handful of highly selected candidates for biological screening. Using this approach, we selected 207 candidates representing a wide range of chemical diversity (for new discovery) or different structures of the same class of compounds (e.g. BBR derivatives) for the screening of novel anti-diabetic drugs using the method established in this study. Apart from obtaining detailed information for the new derivatives from BBR and TP which would allow for detailed structure-activity analysis, our results also confirmed a number of compounds which have been suggested to affect lipid metabolism including tiliroside [29], oxymatrine [30] and FXR ligands [13]. Collectively, these data demonstrate that the screening of the efficacy on cellular lipid accumulation is an effective tool to identify novel compounds with anti-diabetic properties.

Based on the screening data along with the analysis for their drug-like properties, we selected AF and OM for further studies in a model of insulin resistant induced by chronic high fat feeding and hepatic steatosis induced by high fructose feeding, respectively. AF is an active ingredient found in herbal remedies (e.g. Paeonia radix) with a good oral absorption property and pharmacokinetics in vivo [31]. AF is widely used in TCM for its antibiotic, anti-inflammatory and anti-allergic properties with little adverse effects [32,33]. In addition, AF has been reported to lower blood glucose in hyperglycaemic mouse models without increasing pancreatic insulin secretion [34]. We found that AF was able to attenuate the increased body weight gain, visceral adiposity and glucose intolerance induced by chronic high fat feeding without significant effects on food intake, indicating its therapeutic potential for treatment of obesity and diabetes. We further tested the potential
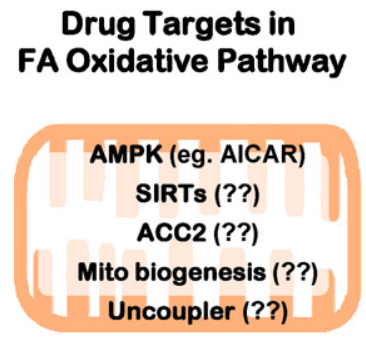

\section{Drug Targets in Lipogenic Pathway}

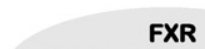

SREBP-1c (eg. Betulin) PPAR $\gamma$ (eg. RSG)

ACC (eg. TOFA)

FAS (eg. C75, Ceru) SCD1 (??)

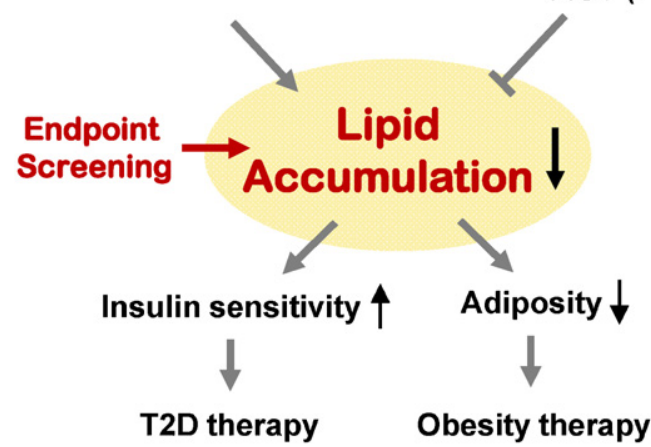

Fig. 7. Illustration of the screening on lipid accumulation for identification of antidiabetic and anti-obese drugs. As excess lipid accumulation is a major cause of insulin resistance in T2D and obesity, drugs that eliminate excess lipid accumulation (in liver and muscle) can improve glucose homeostasis by reversing lipid-induced insulin resistance. Similarly, reducing lipid accumulation in fat tissue can alleviate obesity. The present study has established a HTP assay in cells to determine TG accumulation as a phenotypic screening tool for the identification of new anti-diabetic and anti-obese drugs. This screening assay is capable of capturing drugs that act at various cellular targets in the upstream pathways of lipid metabolism which either increase FA oxidation (energy expenditure) or inhibit de novo lipogenesis. 
application of our screening system by examining another identified lead OM in HFru-fed mice and confirmed its efficacy in reducing body weight gain, hepatosteatosis and glucose intolerance. This may contribute to its reported efficacy for liver fibrosis [35] because hepatosteatosis is an early event of steatohepatitis [36] which could result in liver fibrosis. Taken together, our in vivo data further support the use of the method established in the present study for the identification of new antidiabetic, -obesity and -hepatosteatotic drugs.

In summary, the present study established a new assay to determine TG accumulation in 3T3-L1 cells as a reliable phenotypic screening tool capable of identifying new drugs with anti-obese and anti-diabetic properties by reducing lipid accumulation via different mechanisms. As illustrated in Fig. 7, our results provide the proof of concept for the use of this approach which could be extended to other cell types (such as HepG2 hepatocytes or L6 myocytes) to examine the effect on cell-specific lipid metabolism and serve as a base for further investigation of the effect on FA oxidation, uptake, synthesis and transport.

\section{Authors' contributions}

Conceived and designed the experiments done by JMY, XYZ, JX and CLX. The experiments performed by XZ, XYZ JMY, SMHC. Data analysed by JMY, XZ, XYZ and JCM. JMY, JX and CLX contributed reagents/materials/analysis tools. JMY, XZ, XYZ, SMHC and JCM wrote the paper.

\section{Conflict of interest}

The authors declare that they have no conflict of interest in this study.

\section{Acknowledgements}

This study was supported by the National Health and Medical Research Council of Australia Program Grant (535921 allocation to JMY), Australian Research Council (DP 110102396 to JMY) and the National Natural Science Foundation of China (81173470 to JX).

\section{Appendix A. Supplementary data}

Supplementary data associated with this article can be found, in the online version, at http://dx.doi.org/10.1016/j.bcp.2012.07.003.

\section{References}

[1] Wild S, Roglic G, Green A, Sicree R, King H. Global prevalence of diabetes: estimates for the year 2000 and projections for 2030. Diabetes Care 2004;27:1047-53.

[2] Ellis BA, Poynten A, Lowy AJ, Furler SM, Chisholm DJ, Kraegen EW, et al. Longchain acyl-CoA esters as indicators of lipid metabolism and insulin sensitivity in rat and human muscle. Am J Physiol 2000;279:E554-60.

[3] Ye JM, Frangioudakis G, Iglesias MA, Furler SM, Ellis B, Dzamko N, et al. Prior thiazolidinedione treatment preserves insulin sensitivity in normal rats during acute fatty acid elevation: role of the liver. Endocrinology 2002;143:4527-35.

[4] Yu C, Chen Y, Cline GW, Zhang D, Zong H, Wang Y, et al. Mechanism by which fatty acids inhibit insulin activation of insulin receptor substrate-1 (IRS-1)associated phosphatidylinositol 3-kinase activity in muscle. J Biol Chem 2002;277:50230-36.

[5] Schmitz-Peiffer C, Craig DL, Biden TJ. Ceramide generation is sufficient to account for the inhibition of the insulin-stimulated PKB pathway in C2C12 skeletal muscle cells pretreated with palmitate. J Biol Chem 1999;274:24202-10.

[6] Boden G, She P, Mozzoli M, Cheung P, Gumireddy K, Reddy P, et al. Free fatty acids produce insulin resistance and activate the proinflammatory nuclear factor-kappaB pathway in rat liver. Diabetes 2005;54:3458-65.

[7] Zhang BB, Zhou G, Li C. AMPK: an emerging drug target for diabetes and the metabolic syndrome. Cell Metab 2009;9:407-16.

[8] Loftus TM, Jaworsky DE, Frehywot GL, Townsend CA, Ronnett GV, Lane MD, et al. Reduced food intake and body weight in mice treated with fatty acid synthase inhibitors. Science 2000;288:2379-81.
[9] Wu M, Singh SB, Wang J, Chung CC, Salituro G, Karanam BV, et al. Antidiabetic and antisteatotic effects of the selective fatty acid synthase (FAS) inhibitor platensimycin in mouse models of diabetes. Proc Natl Acad Sci U S A 2011;108:5378-83.

[10] Tang JJ, Li JG, Qi W, Qiu WW, Li PS, Li BL, et al. Inhibition of SREBP by a small molecule, betulin, improves hyperlipidemia and insulin resistance and reduces atherosclerotic plaques. Cell Metab 2011;13:44-56.

[11] Schumann K. Ullmann's encyclopedia of industrial chemistry; 2005.

[12] Tan MJ, Ye JM, Turner N, Hohnen-Behrens C, Ke CQ Tang CP, et al. Antidiabetic activities of triterpenoids isolated from bitter melon associated with activation of the AMPK pathway. Chem Biol 2008;15:263-73.

[13] Zhao W, Gu Q, Wang L, Ge H, Li J, Xu J. Three-dimensional pharmacophore modeling of liver-X receptor agonists. J Chem Inf Model 2011:51:2147-55.

[14] Turner N, Li JY, Gosby A, To SW, Cheng Z, Miyoshi H, et al. Berberine and its more biologically available derivative, dihydroberberine, inhibit mitochondrial respiratory complex I: a mechanism for the action of berberine to activate AMPactivated protein kinase and improve insulin action. Diabetes 2008;57: 1414-8.

[15] Kemper JK, Xiao Z, Ponugoti B, Miao J, Fang S, Kanamaluru D, et al. FXR acetylation is normally dynamically regulated by p300 and SIRT1 but constitutively elevated in metabolic disease states. Cell Metab 2009;10:392-404.

[16] Folch J, Lees M, Sloane Stanley GH. A simple method for the isolation and purification of total lipids from animal tissues. J Biol Chem 1957;226:497-509.

[17] Kinkel AD, Fernyhough ME, Helterline DL, Vierck JL, Oberg KS, Vance TJ, et al. Oil red-O stains non-adipogenic cells: a precautionary note. Cytotechnology 2004;46:49-56.

[18] Ren LP, Chan SM, Zeng XY, Laybutt DR, Iseli TJ, Sun RQ et al. Differing endoplasmic reticulum stress response to excess lipogenesis versus lipid oversupply in relation to hepatic steatosis and insulin resistance. PLoS ONE 2012;7:e30816.

[19] Iglesias MA, Furler SM, Cooney GJ, Kraegen EW, Ye JM. AMP-activated protein kinase activation by AICAR increases both muscle fatty acid and glucose uptake in white muscle of insulin-resistant rats in vivo. Diabetes 2004;53:1649-54.

[20] Huang SL, Yu RT, Gong J, Feng Y, Dai YL, Hu F, et al. Arctigenin, a natural compound, activates AMP-activated protein kinase via inhibition of mitochondria complex I and ameliorates metabolic disorders in ob/ob mice. Diabetologia 2011:55:1469-81.

[21] Tappy L, Le KA. Metabolic effects of fructose and the worldwide increase in obesity. Physiol Rev 2010;90:23-46.

[22] Lehmann JM, Moore LB, Smith-Oliver TA, Wilkison WO, Willson TM, Kliewer SA. An antidiabetic thiazolidinedione is a high affinity ligand for peroxisome proliferator-activated receptor gamma (PPAR $\gamma$ ). J Biol Chem 1995;270: 12953-56.

[23] Juan YC, Tsai WJ, Lin YL, Wang GJ, Cheng JJ, Yang HY, et al. The novel antihyperglycemic effect of Paeoniae radix via the transcriptional suppression of phosphoenopyruvate carboxykinase (PEPCK). Phytomedicine 2010;17:626-34.

[24] Mao YM, Zeng MD, Lu LG, Wan MB, Li CZ, Chen CW, et al. Capsule oxymatrine in treatment of hepatic fibrosis due to chronic viral hepatitis: a randomized, double blind, placebo-controlled, multicenter clinical study. World J Gastroenterol 2004; 10:3269-73.

[25] Boden G. Role of fatty acids in the pathogenesis of insulin resistance and NIDDM. Diabetes 1997;46:3-10.

[26] Huang D, Gu Q, Ge H, Ye J, Salam NK, Hagler A, et al. On the value of homology models for virtual screening: discovering hCXCR3 antagonists by pharmacophore-based and structure-based approaches. J Chem Inf Model 2012;52: 1356-66.

[27] Ruderman NB, Saha AK, Kraegen EW. Minireview: malonyl CoA, AMP-activated protein kinase, and adiposity. Endocrinology 2003;144:5166-71.

[28] Li Y, Xu S, Mihaylova MM, Zheng B, Hou X, Jiang B, et al. AMPK phosphorylates and inhibits SREBP activity to attenuate hepatic steatosis and atherosclerosis in diet-induced insulin-resistant mice. Cell Metab 2011;13:376-88.

[29] Qiao W, Zhao C, Qin N, Zhai HY, Duan HQ. Identification of trans-tiliroside as active principle with anti-hyperglycemic, anti-hyperlipidemic and antioxidant effects from Potentilla chinesis. J Ethnopharmacol 2011;135:515-21.

[30] Hu ST, Tang Y, Shen YF, Ao HH, Bai J, Wang YL, et al. Protective effect of oxymatrine on chronic rat heart failure. J Physiol Sci 2011;61:363-72.

[31] Li YF, Wang M, Wang XY, Yu HS, Kang LP, Ma BP, et al. Pharmacokinetic properties of albiflorin and paeoniflorin after oral administration of pure compound, Radix Paeoniae alba extract and danggui-shaoyao-san extract to rats. J Asian Nat Prod Res 2011;13:117-27.

[32] Zhang HJ, Cheng YY. An HPLC/MS method for identifying major constituents in the hypocholesterolemic extracts of Chinese medicine formula 'Xue-Fu-ZhuYu decoction'. Biomed Chromatogr 2006;20:821-6.

[33] Wu H, Zhu Z, Zhang G, Zhao L, Zhang H, Zhu D, et al. Comparative pharmacokinetic study of paeoniflorin after oral administration of pure paeoniflorin, extract of Cortex Moutan and Shuang-Dan prescription to rats. J Ethnopharmacol 2009;125:444-9.

[34] Hsu FL, Lai CW, Cheng JT. Antihyperglycemic effects of paeoniflorin and 8debenzoylpaeoniflorin, glucosides from the root of Paeonia lactiflora. Planta Med 1997;63:323-5.

[35] Xing Y, Yan F, Liu Y, Zhao Y. Matrine inhibits 3T3-L1 preadipocyte differentiation associated with suppression of ERK1/2 phosphorylation. Biochem Biophys Res Commun 2010;396:691-5.

[36] Neuschwander-Tetri BA. Hepatic lipotoxicity and the pathogenesis of nonalcoholic steatohepatitis: the central role of nontriglyceride fatty acid metabolites. Hepatology 2010;52:774-88. 